Check for updates

Cite this: RSC Adv., 2019, 9, 4028

\title{
Effect of molecular weight distribution of PSSA on electrical conductivity of PEDOT:PSS
}

\author{
Jooyoung Kim, Chanil Park, Soeun Im, Hongjoo Lee and Jung Hyun Kim (DD *
}

Poly(3,4-ethylenedioxythiophene):poly(styrenesulfonate) (PEDOT:PSS) is the most successful conductive polymer. In this study, we investigated the electrical properties of PEDOT:PSS prepared using poly(styrenesulfonic acid) (PSSA) having different molecular weight distributions. Herein PSSA with different molecular weight distributions were successfully polymerized by free radical polymerization and atomtransfer radical polymerization (ATRP). Polydispersity index values of PSSA obtained by the free radical process and ATRP process were 2.3-2.8 and 1.2-1.6 respectively. The electrical conductivity of PEDOT:PSS was enhanced from $376 \mathrm{~S} \mathrm{~cm}^{-1}$ (prepared using free radical PSSA) to $422 \mathrm{~S} \mathrm{~cm}^{-1}$ (prepared using ATRP PSSA) when PSSA of Mn $35000 \mathrm{~g} \mathrm{~mol}^{-1}$ PSSA was used and was enhanced from $234 \mathrm{~S} \mathrm{~cm}^{-1}$ (prepared using free radical PSSA) to $325 \mathrm{~S} \mathrm{~cm}^{-1}$ (prepared using ATRP PSSA) when PSSA of Mn $55000 \mathrm{~g} \mathrm{~mol}^{-1}$ was used, by a factor of $15-30 \%$. The greater the regularity of PSSA, the greater the packing density of PEDOT:PSS and consequently, charge carrier density. The improvement of packing density of PEDOT:PSS was confirmed by improvement in crystallinity of PEDOT:PSS by X-ray diffraction (XRD) analysis.

Received 3rd December 2018 Accepted 13th January 2019

DOI: $10.1039 / \mathrm{c} 8 \mathrm{ra09919g}$

rsc.li/rsc-advances study, they used two different sulfonated polyimide with different molecular weight distribution. ${ }^{7}$ However this study was not for molecular weight distribution of PSSA. To investigate effect of molecular weight distribution of PSSA, we had to find more sophisticated polymer synthesis method.

PSSA is generally polymerized by conventional free radical polymerization, because it is a simple, convenient and costeffective process that does not involve complicated refining. Unlike conventional free radical processes, controlled processes like atom-transfer radical polymerization (ATRP) and reversible addition fragmentation chain transfer (RAFT) polymerization enables the formation of polymers with uniform chain lengths, because of the intrinsic character of the ATRP process. As the name suggests, the atom transfer step is key in the ATRP process and it enables the polymer to have uniform chain length and structure. ${ }^{8-18}$

In this study, we investigated the electrical properties of PEDOT:PSS especially, electrical conductivity prepared using PSSA having different molecular weight distributions. To confirm this hypothesis, we prepared PSSA having different molecular weight distributions. Generally, PSSA polymerized by a free radical process has polydispersity index (PDI) over 2.3. By narrowing down PDI of PSSA under 1.5, we anticipated improvement of electrical conductivity of PEDOT:PSS. The greater the regularity of PSSA, the greater is packing density of PEDOT:PSS and consequently, charge carrier density. The improvement of packing density of PEDOT:PSS was confirmed by improvement of crystallinity of PEDOT:PSS by X-ray diffraction (XRD) analysis.

Conventional PSSA polymerized by a free radical process has polydispersity index (PDI) of 2.3-2.8, while PSSA polymerized by ATRP has PDI of 1.2-1.6. The electrical conductivity of PEDOT:PSS
Department of Chemical Biomolecular Engineering, Yonsei University, 13 Shinchon-Dong, Seodaemoon-Gu, Seoul 120-749, South Korea. E-mail: jayhkim@ yonsei.ac.kr; Fax: +822312 0305; Tel: +82221237633 
was enhanced from $376 \mathrm{~S} \mathrm{~cm}^{-1}$ (prepared using free radical PSSA) to $422 \mathrm{~S} \mathrm{~cm}^{-1}$ (prepared using ATRP PSSA) when PSSA of Mn $35000 \mathrm{~g} \mathrm{~mol}^{-1}$ PSSA and enhanced from $234 \mathrm{~S} \mathrm{~cm}^{-1}$ (prepared using free radical PSSA) to $325 \mathrm{~S} \mathrm{~cm}^{-1}$ (prepared using ATRP PSSA) when PSSA of Mn $55000 \mathrm{~g} \mathrm{~mol}^{-1}$ PSSA by a factor of $15-30 \%$. We investigated these phenomena for improving crystallinity and the primary doping level of PEDOT by using X-ray diffraction (XRD) analysis, UV-vis spectrometry and Raman spectrometry.

\section{Experimental}

\section{Materials}

Styrenesulfonic acid sodium salt hydrate (NaSS, $\geq 99.5 \%$ ), ethyl $\alpha$ bromo-isobutyrate $\left(\mathrm{C}_{6} \mathrm{H}_{11} \mathrm{BrO}_{2}, \geq 98.0 \%\right), 2,2^{\prime}$-bipyridyl $\left(\mathrm{C}_{10} \mathrm{H}_{8}-\mathrm{N}_{2}\right.$, $\geq 99.0 \%)$, copper(I) bromide (CuBr, $\geq 98.0 \%)$, 3,4-ethylenedioxythiphene (EDOT, $\geq 97 \%)$ sodium persulfate $\left(\mathrm{Na}_{2} \mathrm{~S}_{2} \mathrm{O}_{8}\right.$, $\geq 99.0 \%$ ), iron(III) sulfate $\left(\mathrm{Fe}_{2}\left(\mathrm{SO}_{4}\right)_{3}, 97 \%\right)$, dimethyl sulfoxide (DMSO), deuterium oxide (D2O, 99.5 atom\%), methanol $\left(\mathrm{CH}_{3} \mathrm{OH}\right.$, $\geq 99.0 \%$ ) cation exchange resin, anion exchange resin were purchased from Sigma Aldrich Co., Yongin-Si, Gyeonggi-do, Korea. All reagents were used as received without further purification. Distilled deionized (DDI) water was used in all the experiments.

\section{Polymerization of PSSA via free radical process and ATRP process}

For free radical polymerization of PSSA, NaSS (22.2 g, $0.1077 \mathrm{~mol})$ was dissolved in DDI water (190 g) in an inert argon atmosphere at $80{ }^{\circ} \mathrm{C}$. Sodium persulfate $(0.69 \mathrm{~g}, 0.00289 \mathrm{~mol})$ initiator, dissolved in $4 \mathrm{~mL}$ DDI, was added to a $250 \mathrm{~mL}$ double-jacketed glass reactor at $25{ }^{\circ} \mathrm{C}$ and sonicated for $20 \mathrm{~min}$ to obtain sufficient dissolution in the water bath. Stirring speed was maintained at $350 \mathrm{rpm}$ during the reaction. After reaction time of $12 \mathrm{~h}$, cation exchange resin was added to the reactor to remove the residual initiator and any by-products of the reaction.

For PSSA polymerization via ATRP process, (NaSS) (35.24 g, $0.153 \mathrm{~mol})$ and ethyl $\alpha$-bromo-isobutyrate $(0.1 \mathrm{~g}, 0.51 \mathrm{mmol})$ as an alkyl halide initiator were dissolved in DDI water $(204 \mathrm{~g})$ and methanol $(17 \mathrm{~g})$ in an inert argon atmosphere at $10{ }^{\circ} \mathrm{C} . \mathrm{CuBr}$ $(0.15 \mathrm{~g}, 0.001 \mathrm{~mol})$ catalyst and 2,2'-bipyridyl $(0.31 \mathrm{~g}, 0.002 \mathrm{~mol})$ ligand were dissolved in methanol $(4 \mathrm{~mL})$ at $25{ }^{\circ} \mathrm{C}$ added to the reactor and sonicated for $20 \mathrm{~min}$ to obtain sufficient dissolution in the water bath. Stirring speed were maintained at $350 \mathrm{rpm}$ during reaction. After reaction time of $12 \mathrm{~h}$, cation exchange resin and anion exchange resin were added to the reactor to remove the residual initiator and any residual by-products of the reaction. PSSA samples obtained by the two above-mentioned method were dried to eliminate water and methanol by heating at $50{ }^{\circ} \mathrm{C}$ to minimize the influence of thermal energy.

\section{Polymerization of PEDOT:PSS using 2 different PSSA samples}

PEDOT:PSS was polymerized via oxidative polymerization. First, $0.84 \mathrm{~g}$ of PSSA was dissolved in $73 \mathrm{~g}$ of DDI water and stirred at $400 \mathrm{rpm}$ until sufficient dissolution in an inert argon atmosphere at $10{ }^{\circ} \mathrm{C}$. Then, (EDOT) was added to the reactor. $\mathrm{Fe}_{2}\left(\mathrm{SO}_{4}\right)_{3}(0.0053 \mathrm{~g})$ in DDI water $(3 \mathrm{~g})$ and $\mathrm{Na}_{2} \mathrm{~S}_{2} \mathrm{O}_{8}(0.772 \mathrm{~g})$ in DDI water ( $2 \mathrm{~g})$ were sequentially added to the reactor. After reaction time of $24 \mathrm{~h}$, cation exchange resins and anion exchange resins were added the reactor to remove the residual initiator and any residual by-products of the reaction.

\section{Characterization}

Structural characterization of both PSSA samples, made via free radical process and ATRP process, were performed by ${ }^{1} \mathrm{H}$ nuclear magnetic resonance (NMR) spectroscopy (Bruker Biospin, Advanced III, Seongnam-si, Korea). Molecular weight and its distribution in both PSSA samples made via free radical process and ATRP process were measured by gel permeation chromatography (GPC) (YL instruments, ACME9000 equipped with a series of Waters columns; HR4, HR3, HR2 and HR1; Anyang-si, Korea). Before analysis, PSSA samples were dried at $50{ }^{\circ} \mathrm{C}$ to eliminate water for minimizing the effect of thermal energy. UV-visible absorption spectra were obtained on a UV-vis spectrometer (Jasco, Jasco V-700, Seoul, Korea). Raman spectra were measured by Raman spectrometer (LabRam Aramis, Horriba Jobin Yvon, Seoul, Korea). Dispersive stability of PEDOT:PSS were measured by a zeta potential analyzer (Malvern instrument, Zetasize Nano Zs, Seongnam-si, Korea). Crystallinity of PEDOT:PSS were measured by X-ray diffraction analyzer (Rigaku, SmartLab, Seoul, Korea). The sheet resistance were measured by four point probe method (Napson, RT-70V/RG5, Seoul, Korea) and the film thicknesses were measured by a surface profiler (Bruker, DektakXT Stylus Profiler, Seongnam-si, Korea). The elemental composition of the PEDOT:PSS film surface was analysed by X-ray photoelectron spectroscopy (Thermo UK, K-alpha, Seoul, Korea).

Electrical conductivity of PEDOT:PSS were measured following procedure. PEDOT:PSS solution was coated on the bare glass substrate by spin coater with 300, 500, 700, $900 \mathrm{rpm}$ respectively. After coating, we annealed the PEDOT:PSS coated glass in drying oven at $120^{\circ} \mathrm{C}$ for 2 minutes. Sheet resistance were measured using four point probe method. Then the thickness of PEDOT:PSS were measured using surface profiler by 4 times. We averaged 4 times measurement thickness values and used the averaged thickness value. The electrical conductivity were calculated inverse value of sheet resistance divided by sheet thickness.

\section{Results and discussion}

\section{General opinions on factors affected on electrical conductivity of PEDOT:PSS}

As per Ohm's law, current can be obtained using the following equation (current is generally denoted by $i$ and electromotive force is generally denoted by $E$ ).

$$
i=\sigma E=E / \rho
$$

Resistivity is a characteristic that quantifies how much a given material opposes the flow of current, i.e. current flows easily when the resistance is low. The resistivity is generally denoted by $\rho$ and electrical conductivity, i.e. the inverse of resistivity is generally denoted by $\sigma$. Conductivity can be expressed by the following equation (sheet resistance is generally denoted by $R_{\mathrm{s}}$ and sheet thickness is generally denoted by $t$ ). 

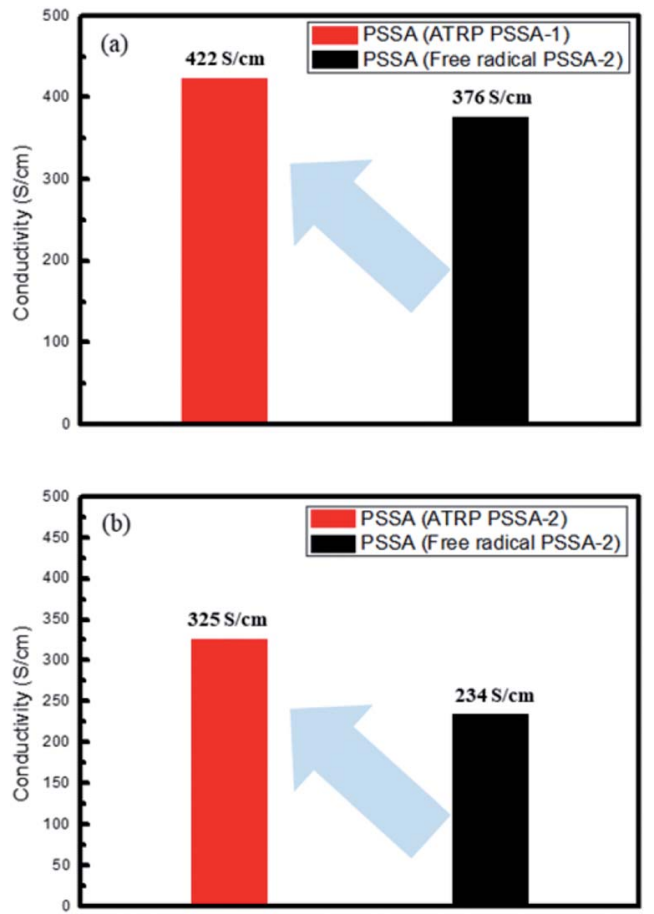

Fig. 1 PEDOT:PSS (PSSA-1 (a)/PSSA-2 (b)) electrical conductivity.

Table 1 Average electrical conductivity of PEDOT:PSS

\begin{tabular}{ll}
\hline Description & Average conductivity $\left(\mathrm{S} \mathrm{cm}^{-1}\right)$ \\
\hline PEDOT:PSS (ATRP PSSA-1) & 422 \\
PEDOT:PSS (free radical PSSA-1) & 376 \\
PEDOT:PSS (ATRP PSSA-2) & 325 \\
PEDOT:PSS (free radical PSSA-2) & 234
\end{tabular}

$$
\rho=R_{\mathrm{s}} t, \sigma=1 / \rho
$$

When materials are electrically charged, carrier works under electrical field. When the carrier is electron, the material is termed as electrical-conducting and when the carrier is ion, it can be termed as ion-conducting. In this study, we deal with electrical conduction. Electrical conductivity can be expressed by the following equation (charge carrier density is generally denoted by $n$, elemental charge is generally denoted by $e$ and charge carrier mobility is generally denoted by $\mu$ ).

$$
\sigma=n e \mu
$$

For PEDOT:PSS, only holes participate in charge transportation, because free electrons immediately recombine at the oxidized PEDOT. To obtain high electrical conductivity for PEDOT:PSS, the condition of high density and mobility of charge carriers must be satisfied. In this study, charge carrier density is thought by crystallinity of PEDOT:PSS from XRD measurement and charge carrier mobility of PEDOT:PSS is thought by getting easily doped from UV-visible spectroscopy and Raman spectroscopy. The greater the crystallinity of PEDOT:PSS, the greater is its packing density and consequently, charge carrier density.

Conventional PSSA polymerized by a free radical process has polydispersity index (PDI) of 2.3-2.8, while PSSA polymerized by ATRP has PDI of 1.2-1.6. The electrical conductivity of PEDOT:PSS was enhanced from $376 \mathrm{~S} \mathrm{~cm}^{-1}$ (prepared using free radical PSSA) to $422 \mathrm{~S} \mathrm{~cm}^{-1}$ (prepared using ATRP PSSA) when PSSA of $\mathrm{Mn}=35000 \mathrm{~g} \mathrm{~mol}^{-1}$ PSSA and enhanced from $234 \mathrm{~S} \mathrm{~cm}^{-1}$ (prepared using free radical PSSA) to $325 \mathrm{~S} \mathrm{~cm}^{-1}$ (prepared using ATRP PSSA) when PSSA of Mn = $55000 \mathrm{~g} \mathrm{~mol}^{-1}$ PSSA by a factor of $15-30 \%$ (Fig. 1 and Table 1 ).

\section{Polymerization of PSSA with narrow molecular weight distribution via ATRP and with broad molecular weight distribution via free radical process}

The ATRP process involves relatively mild reaction conditions, because this polymerization process is initiated by atoms instead of radicals and reaction temperature is $\sim 10{ }^{\circ} \mathrm{C}$. Due to the characteristics of the reaction mechanism, PSSA prepared by ATRP process has narrow molecular weight distribution (PDI

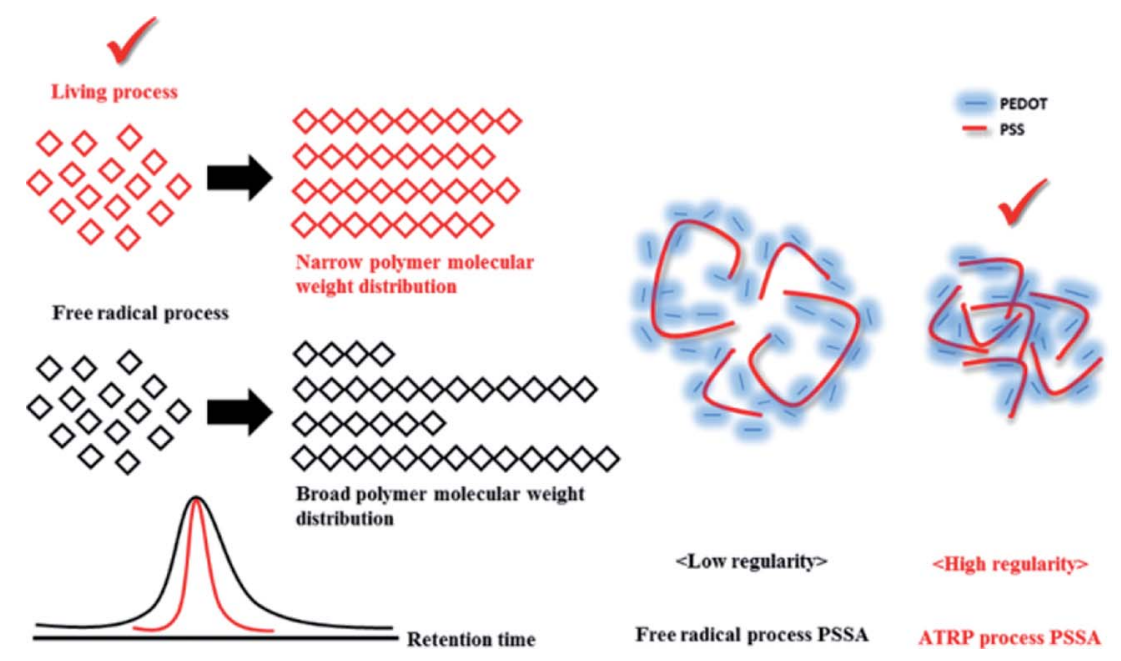

Scheme 1 Schematic illustration of effect of molecular weight distribution of PSSA on electrical conductivity of PEDOT:PSS. 
is under 1.6). On the other hands, the free radical process involves relatively harsh reaction conditions, because the highly reactive radical molecules must be generated by thermal cleavage, and the reaction temperature is above $80{ }^{\circ} \mathrm{C}$. Based on these differences of the intrinsic reaction mechanism, PSSA samples prepared by the two processes have different molecular weight distributions. Generally, PSSA obtained from the free radical process and the ATRP process have PDI values greater than 2.0, and less than 1.6 respectively. (Scheme 1) To check the differences in molecular weight distribution between PSSA obtained by the two processes, GPC measurement was implemented. Before measurement, we calibrated the GPC instrument using standard GPC samples with different molecular weights $\left(\mathrm{Mn}=37\right.$ 500/60 000/210, 400/740, 500) ${ }^{\mathbf{1 9 , 2 0}}$ (Fig. 2 and Table 2).

${ }^{1} \mathrm{H}$ NMR spectroscopy was used for structural characterization of PSSA samples polymerized via free radical process and ATRP process, $\mathrm{D}_{2} \mathrm{O}$ was used as the NMR solvent to dissolve PSSA samples. It was found that PSSA polymerized by the two different processes have identical structures ${ }^{21-23}$ (Fig. 3).

We prepared PSSA of two molecular weight group: one with $\mathrm{Mn}=33000-35000 \mathrm{~g} \mathrm{~mol}^{-1}$ and the other with $\mathrm{Mn}=55000-$ $58000 \mathrm{~g} \mathrm{~mol}^{-1}$. GPC measurement revealed that PSSA polymerized by the ATRP process and the free radical process have PDI values of less than 1.6 and greater than 2.3, respectively. By comparing the PDI values of PSSA prepared by the two processes, we ascertained the differences between the two processes.

\section{Polymerization of PEDOT with PSSA with narrow molecular weight distribution, prepared via the ATRP process and with PSSA with broad molecular weight distribution, prepared via the free radical process}

PSSA acts as a dispersion stabilizer in water and as a counter ion or dopant for PEDOT:PSS in water. To investigate the effect of molecular weight distribution of PSSA on the electrical conductivity of PEDOT:PSS, we performed polymerization of PEDOT using PSSA prepared via the ATRP process and the free radical process. PSSA ratio for EDOT monomer was $250 \mathrm{wt} \%$. The reaction conditions, i.e. reaction temperature, feed rate of purging argon gas for preventing air inflow, agitation speed and reaction time were identical. Before studying the electrical properties of PEDOT:PSS, we checked the dispersive stability of PEDOT:PSS using the zeta potential analyzer. PEDOT:PSS solution was diluted 1 : 100 using DDI water. Both PEDOT:PSS samples had potential values between $-85 \mathrm{mv}$ and $-95 \mathrm{mV}$, and thus showed good dispersive stability. When the absolute value of zeta potential is ranged $50-100 \mathrm{mV}$, the dispersion is considered stable. ${ }^{24}$

We ascertained that PEDOT:PSS obtained using ATRP PSSA had higher electrical conductivity than PEDOT:PSS obtained using free radical PSSA. Higher electrical conductivity originates from the structural regularity of the PEDOT:PSS molecules. Hui et al. stated that entangled PEDOT:PSS chain changed into linear or expanded coil formation by UV-ozone treatment. Accordingly the number of charge trapping defects decreases and the electrical conductivity is enhanced. ${ }^{25}$ ATRP PSSA has more regular molecular weight distribution than free radical
PSSA; therefore, PEDOT:PSS prepared using ATRP PSSA forms more crystalline structure than PEDOT:PSS prepared using free radical PSSA. This was confirmed by analyzing the crystallinity of PEDOT:PSS.
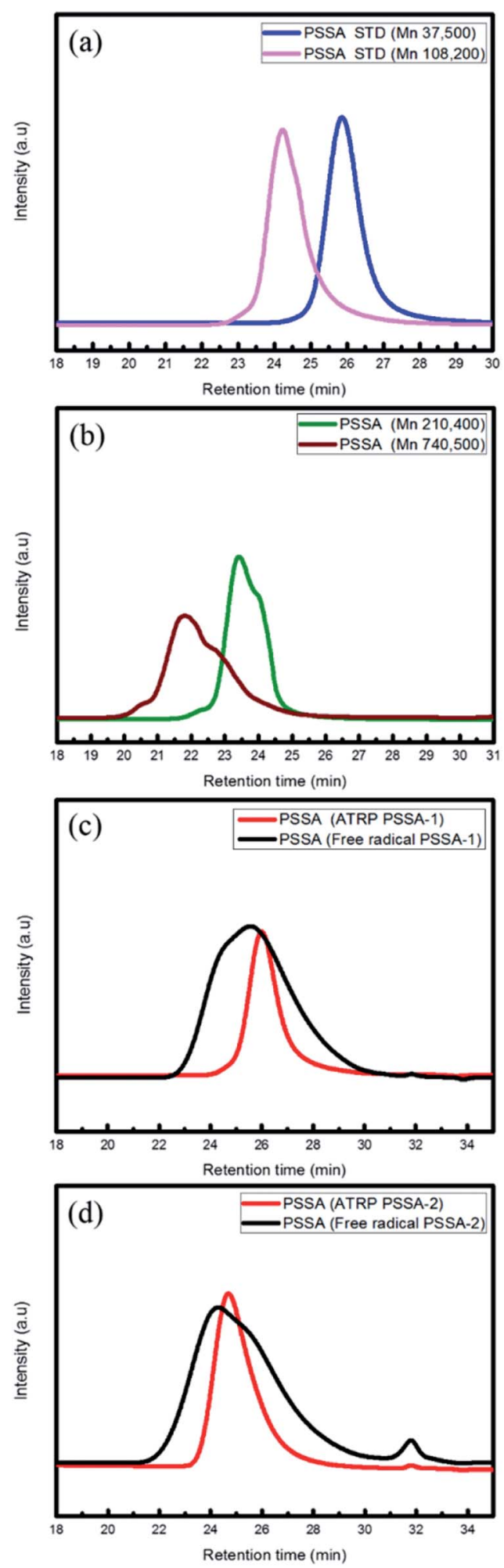

Fig. 2 GPC calibration by PSSA standard sample and measurement of molecular weight of PSSA prepared by the ATRP and free radical processes (PSSA standard (a and b), PSSA-1 (c), PSSA-2 (d)). 
Table 2 PSSA molecular weight data (GPC)

\begin{tabular}{llll}
\hline Description & $\mathrm{Mn}\left(\mathrm{g} \mathrm{mol}^{-1}\right)$ & $\mathrm{Mw}(\mathrm{g} \mathrm{mol}$ & \\
\hline PSSA-1 (ATRP) & 33000 & 40000 & PDI \\
PSSA-1 (free radical) & 35000 & 81000 & 1.21 \\
PSSA-2 (ATRP) & 58000 & 99000 & 2.31 \\
PSSA-2 (free radical) & 55000 & 153000 & 1.65 \\
& &
\end{tabular}

Kim et al. stated that The XRD patterns show characteristic peaks at $2 \theta=3.8^{\circ}, 6.6^{\circ}, 17.7^{\circ}, 25.6^{\circ}$. The low-angle reflections at $2 \theta=3.8^{\circ}$ show the lamellar stacking distance of two distinct alternate ordering of PEDOT and PSS, whereas the high angle reflections at $2 \theta=17.7^{\circ}$ and $25.6^{\circ}$ show the amorphous halo of PSS and the interchain planar ring-stacking distance of
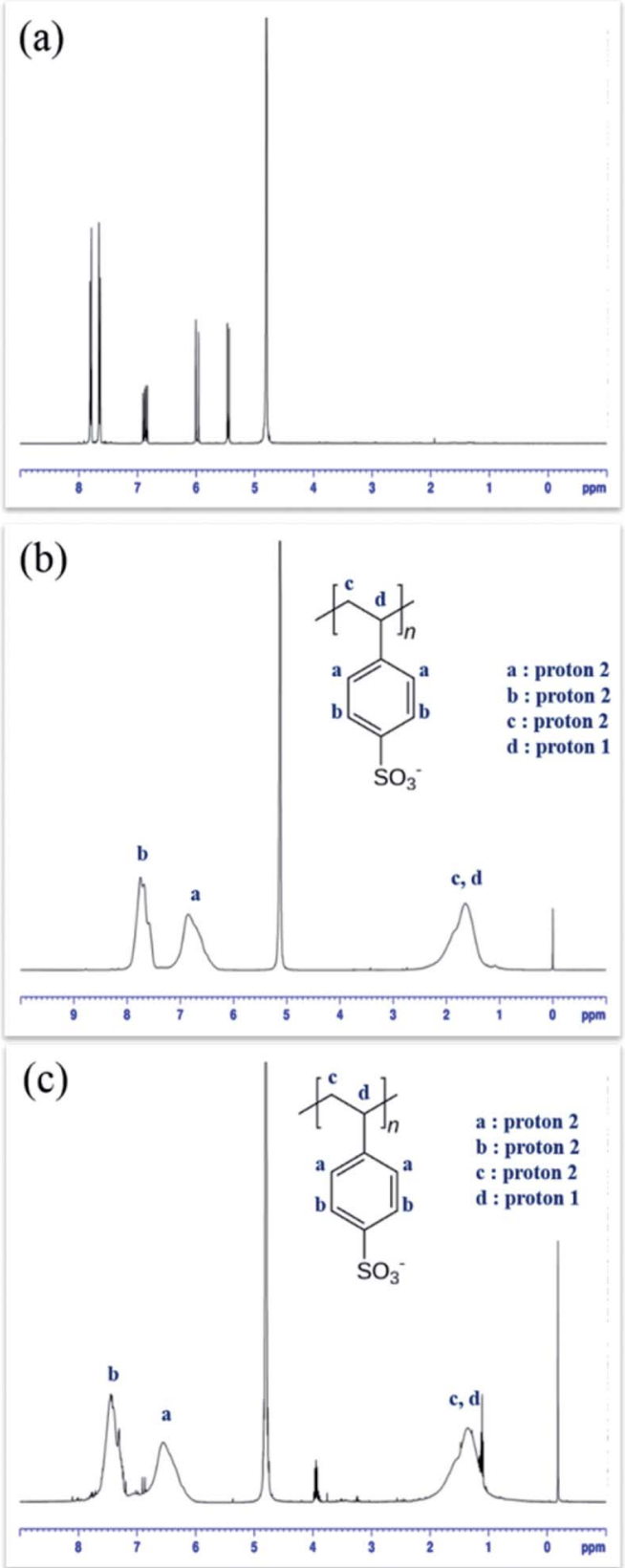

Fig. $3{ }^{1} \mathrm{H}$ NMR spectra of NaSS (a), free radical PSSA (b), ATRP PSSA (c).
PEDOT. ${ }^{26-33}$ XRD spectrum (Fig. 4) revealed that PEDOT and PSS became more crystalline when prepared using ATRP PSSA. At low-angle reflection at $3.8^{\circ}$, PEDOT:PSS using ATRP PSSA shows higher intensity value and At high-angle reflection at $17.7^{\circ}$ and $25.6^{\circ}$, PEDOT:PSS using ATRP PSSA shows lower intensity value than PEDOT:PSS using free radical PSSA.

High electrical conductivity also originates from the primary doping level of PEDOT:PSS prepared by using the more regular PSSA obtained from the ATRP process. To confirm this hypothesis, we performed UV-vis and Raman spectroscopic analyses.

The UV-visible spectra (Fig. 5) showed that with increasing amount of primary dopant (PSSA) in the PEDOT molecules,

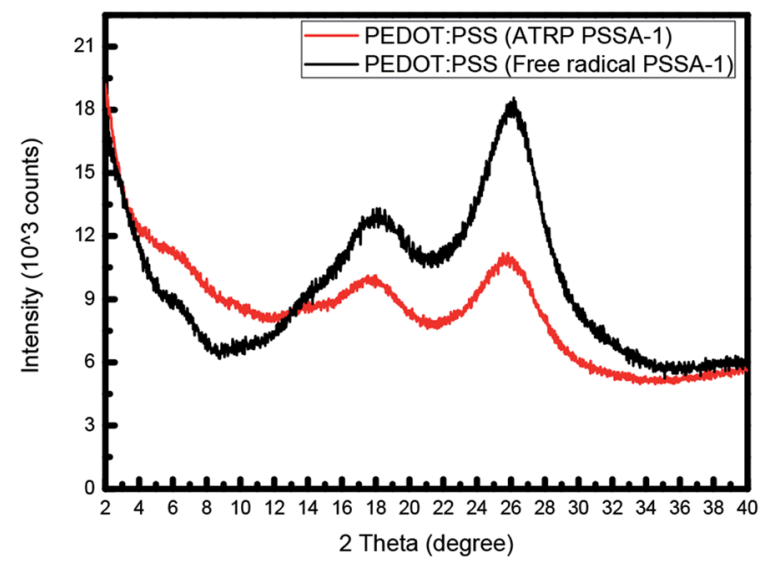

Fig. 4 X-ray diffraction (XRD) spectrum of PEDOT:PSS.
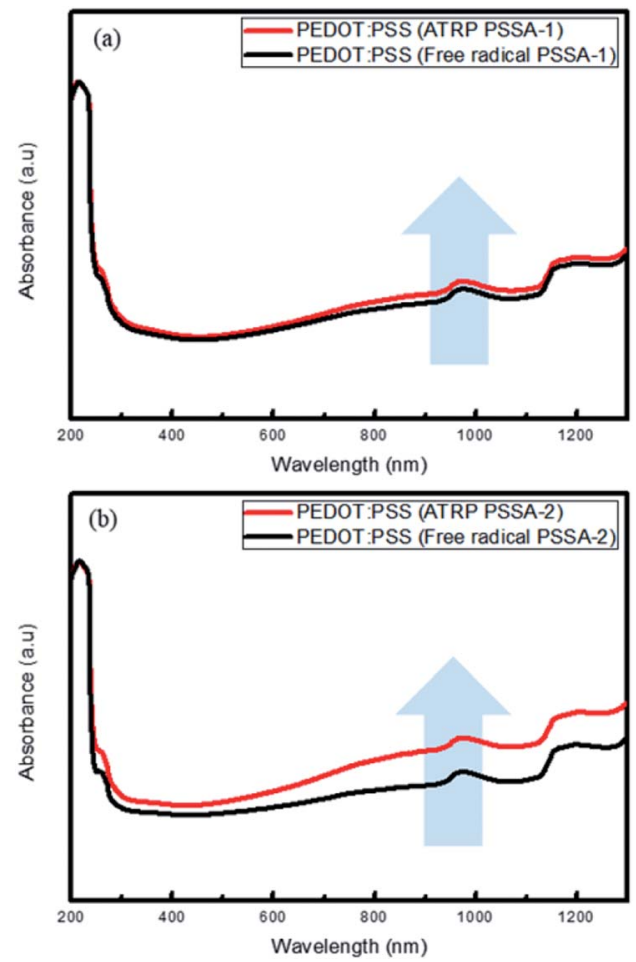

Fig. 5 UV-visible spectra (absorbance mode) of PEDOT:PSS (ATRP) and PEDOT:PSS (free radical) (a) PSSA-1 (b) PSSA-2. 
absorbance increases, especially in the near-IR (NIR) range (800-1400 nm). We ascertained that PEDOT:PSS prepared using ATRP PSSA absorbs more NIR light than that prepared using free radical PSSA. ${ }^{34}$

Raman spectra (Fig. 6) revealed that PEDOT became more crystalline and was stabilized by resonance by transforming from the benzoid structure to the quinoid structure when prepared using ATRP PSSA. The five main peaks observed are attributed to the $\mathrm{C}_{\alpha}-\mathrm{C}_{\beta}$ antisymmetric stretching vibrations (1517 $\left.\mathrm{cm}^{-1}\right), \quad \mathrm{C}_{\alpha}=\mathrm{C}_{\beta} \quad$ symmetric stretching vibrations (1438 $\left.\mathrm{cm}^{-1}\right), \quad \mathrm{C}_{\beta}-\mathrm{C}_{\beta}$ deformations $\left(1373 \mathrm{~cm}^{-1}\right), \quad \mathrm{C}_{\alpha}-\mathrm{C}_{\alpha^{\prime}}$ symmetric stretching vibrations $\left(1263 \mathrm{~cm}^{-1}\right)$ and oxyethylene ring deformation $\left(996 \mathrm{~cm}^{-1}\right)$. The two structures have different $\pi$-conjugations and charge delocalizations. When this phenomenon occurs, the Raman spectra show red shift. (The peak due to PEDOT $\mathrm{C}_{\alpha}=\mathrm{C}_{\beta}$ symmetric stretching shifted to shorter wavenumber, i.e. $1450 \mathrm{~cm}^{-1}$ ) ATRP PSSA have more crystalline structure than free radical PSSA; therefore, ATRP PSSA can easily donate proton to PEDOT. For PSSA-1, the $\mathrm{C}_{\alpha}=$ $\mathrm{C}_{\beta}$ symmetric stretching vibrations were shifted from $1441 \mathrm{~cm}^{-1}$ (free radical) to $1431 \mathrm{~cm}^{-1}$ (ATRP) and PSSA-2, the $\mathrm{C}_{\alpha}=\mathrm{C}_{\beta}$ symmetric stretching vibrations were shifted from $1448 \mathrm{~cm}^{-1}$ (free radical) to $1444 \mathrm{~cm}^{-1}$ (ATRP). ${ }^{35-37}$

Seo et al. investigated the effect of $\mathrm{TiO}_{2}$ nanoparticle addition on the structure of PEDOT:PSS. In this study, $\mathrm{TiO}_{2}$ nanoparticle added PEDOT:PSS shows structural change from benzoid to quinoid in Raman spectroscopy. The benzoid prefer coil conformation structure, while the quinoid prefer linear or expanded-coil structure. ${ }^{38}$ From this study, relatively linear
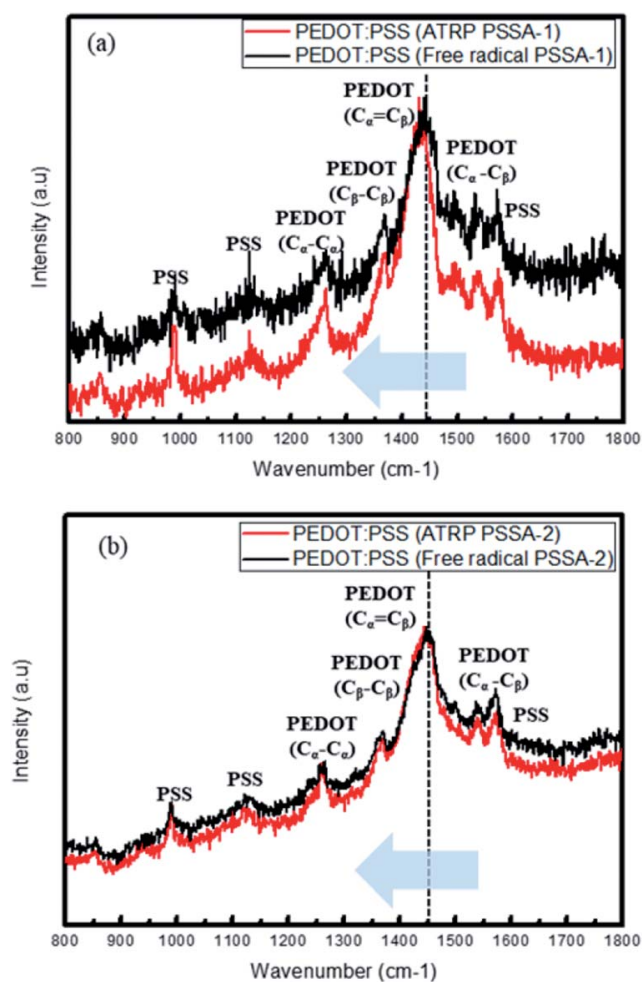

Fig. 6 Raman spectra of PEDOT:PSS (ATRP) and PEDOT:PSS (free radical) (a) PSSA-1 (b) PSSA-2. polymer complex, PEDOT:PSS synthesized from ATRP process shows quinoid structure, while entangled coil polymer complex, PEDOT:PSS synthesized from free radical process shows benzoid structure in Raman spectroscopy.

\section{Conclusions}

We investigated the effect of PSSA with different molecular weight distributions on the electrical conductivity of PEDOT:PSS. Although PSSA plays an important role in the PEDOT:PSS complex as polyanionic polyelectrolyte and a primary dopant, the effect of molecular weight distribution of PSSA was not investigated before. We ascertained that PSSA polymerized via controlled radical processes like ATRP had narrow molecular weight distribution compared to PSSA polymerized via free radical process. We used ethyl $\alpha$-bromo-isobutyrate as an atom transfer agent for the ATRP process. Compared to the free radical process, the ATRP process polymerized PSSA under milder reaction conditions, especially the reaction temperature (free radical process: $80{ }^{\circ} \mathrm{C}$, ATRP process: $10{ }^{\circ} \mathrm{C}$ ). In addition, more reactive radicals initiated the polymerization in free radical process, while less reactive atoms initiated the polymerization in the ATRP process. Therefore, PSSA polymerized via the ATRP process has narrower molecular weight distribution than PSSA polymerized via the free radical process (PDI values obtained using the free radical process and the ATRP process are 2.3-2.8 and 1.2-1.6 respectively). The electrical conductivity of PEDOT:PSS was enhanced from $376 \mathrm{~S} \mathrm{~cm} \mathrm{~cm}^{-1}$ (using free radical PSSA) to $422 \mathrm{~S} \mathrm{~cm}^{-1}$ (using ATRP PSSA) when prepared using PSSA of Mn $=35000 \mathrm{~g} \mathrm{~mol}^{-1}$ and was enhanced from $234 \mathrm{~S} \mathrm{~cm}^{-1}$ (using free radical PSSA) to $325 \mathrm{~S} \mathrm{~cm}^{-1}$ (using ATRP PSSA) when prepared using PSSA of $\mathrm{Mn}=55000 \mathrm{~g} \mathrm{~mol}^{-1}$ PSSA by a factor of $15-30 \%$. XRD analysis confirmed that the enhancement of electrical conductivity originated from the increase in crystallinity. The UVvis and Raman spectra revealed that the enhancement in electrical conductivity was due to the primary doping level and stabilization of the PEDOT structure.

\section{Conflicts of interest}

There are no conflicts to declare.

\section{Acknowledgements}

This work was supported by the Nano Material Technology Development Program through the National Research Foundation of Korea (NRF) funded by the Ministry of Science, ICT \& Future Planning (MSIP, Korea) (NRF-2014M3A7B4050960/ 2014M3A7B4051745). This research was also supported by the Priority Research Centers Program via the National Research Foundation of Korea (NRF) funded by the Ministry of Education, Science and Technology (2009-0093823).

\section{Notes and references}

1 W. Cho, S. Im, S. Kim, S. Kim and J. H. Kim, Polymers, 2016, 8, 189. 
2 C. h. Chen, J. C. LaRue, R. D. Nelson, L. Kulinsky and M. J. Madou, J. Appl. Polym. Sci., 2012, 125, 3134-3141.

3 J. E. McCarthy, C. A. Hanley, L. J. Brennan, V. G. Lambertini and Y. K. Gun'ko, J. Mater. Chem. C, 2014, 2, 764-770.

4 S. Mukherjee, R. Singh, S. Gopinathan, S. Murugan, S. Gawali, B. Saha, J. Biswas, S. Lodha and A. Kumar, ACS Appl. Mater. Interfaces, 2014, 6, 17792-17803.

5 Z. Li, F. Qin, T. Liu, R. Ge, W. Meng, J. Tong, S. Xiong and Y. Zhou, Org. Electron., 2015, 21, 144-148.

6 W. Cho, J. K. Hong, J. J. Lee, S. Kim, S. Kim, S. Im, D. Yoo and J. H. Kim, RSC Adv., 2016, 6, 63296-63303.

7 S. Srisuwan, Y. Ding, D. Mamangun, S. Thongyai, P. Praserthdam and G. A. Sotzing, J. Appl. Polym. Sci., 2013, 128, 3840-3845.

8 C.-K. Choi and Y.-B. Kim, Polym. Bull., 2003, 49, 433-439.

9 P. Iddon, K. Robinson and S. Armes, Polymer, 2004, 45, 759768.

10 J. K. Hong, Y. S. Kim, W. Cho and J. H. Kim, Macromol. Res., 2017, 25, 243-248.

11 M. Kato, M. Kamigaito, M. Sawamoto and T. Higashimura, Macromolecules, 1995, 28, 1721-1723.

12 J.-S. Wang and K. Matyjaszewski, J. Am. Chem. Soc., 1995, 117, 5614-5615.

13 B. Keoshkerian, M. K. Georges and D. Boils-Boissier, Macromolecules, 1995, 28, 6381-6382.

14 Y. Mitsukami, M. S. Donovan, A. B. Lowe and C. L. McCormick, Macromolecules, 2001, 34, 2248-2256.

15 Z. Tang, S. Liu, Z. Wang, S. Dong and E. Wang, Electrochem. Commun., 2000, 2, 32-35.

16 X.-S. Wang, S. Lascelles, R. Jackson and S. Armes, Chem. Commun., 1999, 1817-1818.

17 K. L. Robinson, J. V. Weaver, S. P. Armes, E. D. Marti and F. C. Meldrum, J. Mater. Chem., 2002, 12, 890-896.

18 P. Liu, D. Liang, Z. Tong and X. Liu, Macromolecules, 2002, 35, 1487-1488.

19 R. W. Lewis, R. A. Evans, N. Malic, K. Saito and N. R. Cameron, Polym. Chem., 2017, 8, 3702-3711.

20 K. S. Kang, C. H. Jee, T. Kim, I. Chung and P. Huh, Polym.Plast. Technol. Eng., 2017, 56, 117-122.
21 J. C. Yang, M. J. Jablonsky and J. W. Mays, Polymer, 2002, 43, 5125-5132.

22 W. J. Bae, K. H. Kim, Y. H. Park and W. H. Jo, Chem. Commun., 2003, 2768-2769.

23 S. Hietala, S. L. Maunu and F. Sundholm, Macromolecules, 1999, 32, 788-791.

24 G. Wang, Y. Ding, F. Wang, X. Li and C. Li, J. Colloid Interface Sci., 2008, 317, 199-205.

25 H. Shi, C. Liu, Q. Jiang and J. Xu, Adv. Electron. Mater., 2015, 1, 1500017.

26 N. Kim, S. Kee, S. H. Lee, B. H. Lee, Y. H. Kahng, Y. R. Jo, B. J. Kim and K. Lee, Adv. Mater., 2014, 26, 2268-2272.

27 N. Kim, B. H. Lee, D. Choi, G. Kim, H. Kim, J.-R. Kim, J. Lee, Y. H. Kahng and K. Lee, Phys. Rev. Lett., 2012, 109, 106405.

28 K. Aasmundtveit, E. Samuelsen, L. Pettersson, O. Inganäs, T. Johansson and R. Feidenhans, Synth. Met., 1999, 101, 561-564.

29 N. Murthy and H. Minor, Polymer, 1990, 31, 996-1002.

30 M. V. Fabretto, D. R. Evans, M. Mueller, K. Zuber, P. HojatiTalemi, R. D. Short, G. G. Wallace and P. J. Murphy, Chem. Mater., 2012, 24, 3998-4003.

31 A. Laforgue and L. Robitaille, Macromolecules, 2010, 43, 4194-4200.

32 J. Y. Kim, M. H. Kwon, Y. K. Min, S. Kwon and D. W. Ihm, Adv. Mater., 2007, 19, 3501-3506.

33 M. A. Ali, H. Kim, C. Lee, H. Nam and J. Lee, Synth. Met., 2011, 161, 1347-1352.

34 Y. Wang, C. Zhu, R. Pfattner, H. Yan, L. Jin, S. Chen, F. Molina-Lopez, F. Lissel, J. Liu and N. I. Rabiah, Sci. Adv., 2017, 3, e1602076.

35 Y. Kim, W. Cho, Y. Kim, H. Cho and J. H. Kim, J. Mater. Chem. C, 2018, 6, 8906-8913.

36 J. Y. Oh, M. Shin, J. B. Lee, J.-H. Ahn, H. K. Baik and U. Jeong, ACS Appl. Mater. Interfaces, 2014, 6, 6954-6961.

37 T.-R. Chou, S.-H. Chen, Y.-T. Chiang, Y.-T. Lin and C.-Y. Chao, J. Mater. Chem. C, 2015, 3, 3760-3766.

38 H. Seo, M.-K. Son, N. Itagaki, K. Koga and M. Shiratani, J. Power Sources, 2016, 307, 25-30. 